\title{
Double reporting and second opinion in head and neck pathology
}

\author{
Julia A. Woolgar • Asterios Triantafyllou $\cdot$ Lester D. R. Thompson • Jennifer L. Hunt \\ James S. Lewis Jr. • Michelle D. Williams • Antonio Cardesa • Alessandra Rinaldo • Leon Barnes • \\ Pieter J. Slootweg $\cdot$ Kenneth O. Devaney $\cdot$ Douglas R. Gnepp $\cdot$ William H. Westra $\cdot$ Alfio Ferlito
}

Received: 15 December 2013/Accepted: 31 December 2013/Published online: 17 January 2014

(C) Springer-Verlag Berlin Heidelberg 2014

\section{Introduction: definitions}

This editorial aims to discuss the practice of "double reporting" and "second opinion" diagnosis in routine diagnostic pathology interpretation. It does not encompass reviews performed as part of audit and quality assurance functions, but is from the perspective of experienced head and neck and oral and maxillofacial specialists.

"Double reporting" generally refers to showing a case to one or more colleagues working in the same histopathology unit before issuing a malignant diagnosis [1]. When there is concurrence, the fact that the case has been

This paper was written by members of the International Head and Neck Scientific Group (www.IHNSG.com).

J. A. Woolgar · A. Triantafyllou

Oral Pathology, School of Dentistry, University of Liverpool,

Liverpool, UK

L. D. R. Thompson

Department of Pathology, Woodland Hills Medical Center,

Woodland Hills, CA, USA

J. L. Hunt

Department of Pathology, University of Arkansas for Medical

Sciences, Little Rock, AR, USA

\section{J. S. Lewis Jr.}

Department of Pathology and Immunology and Otolaryngology-

Head and Neck Surgery, Washington University School of

Medicine, St. Louis, MO, USA

M. D. Williams

Department of Pathology, The University of Texas MD

Anderson Cancer Center, Houston, TX, USA

A. Cardesa

Department of Anatomic Pathology, Hospital Clinic,

University of Barcelona, Barcelona, Spain seen by two pathologists may or may not be mentioned in the report, often determined by individual practice, medical-legal environments and relative value units of workload. The final report may be signed by all pathologists who reviewed the case or might simply include a statement that the "case has been reviewed by \{name of reviewer(s)\}, who concur(s) with the diagnosis". In case of a major disagreement between experienced pathologists in the same unit (e.g., benign vs. malignant), additional evaluation should be solicited and the issued diagnosis may be a majority decision. This difference of opinion should be mentioned in the report, with typical examples including cases of melanoma or hematopoietic and lymphoid neoplasia. The practice of "double reporting" varies between

A. Rinaldo · A. Ferlito $(\bowtie)$

ENT Clinic, Piazzale S. Maria della Misericordia,

University of Udine, 33100 Udine, Italy

e-mail: a.ferlito@uniud.it

L. Barnes

Department of Pathology, University of Pittsburgh School of Medicine, Pittsburgh, PA, USA

P. J. Slootweg

Department of Pathology, Radboud University Nijmegen

Medical Centre, Nijmegen, The Netherlands

K. O. Devaney

Department of Pathology, Allegiance Health, Jackson, MI, USA

D. R. Gnepp

Department of Pathology, Warren Alpert School of Medicine at

Brown University, Rhode Island Hospital, Providence, RI, USA

W. H. Westra

Departments of Pathology and Otolaryngology-Head

and Neck Surgery, The Johns Hopkins Medical Institutions,

Baltimore, MD, USA 
diagnostic histopathology units worldwide, from nonexistent to voluntary to mandatory. Nevertheless, increasing risk from medical litigation may eventually tip the scales towards mandatory double reporting as a standard protocol for subsets of pathology diagnosis, such as malignant lesions. "Double reporting" may also be applied to unusual, rare, and difficult diagnoses, including benign lesions. The variations in practices have made comparisons between institutions difficult, with very little reported literature. Nevertheless, a study involving 45 laboratories in the USA reported a median rate of review of about 1 in 12 cases, with malignancy and difficult diagnosis being the primary reported reasons for review [2]. The study documented that head and neck lesions accounted for $4.1 \%$ of cases with "double reporting", while three-fifths were focused on gastrointestinal tract, breast, skin and female genital tract lesions [2].

Referring a case for a "second opinion" implies the traditional, formal approach of sending a case to an external, recognized specialist department or individual pathologist with experience and expertise in a particular field. It often involves rare or difficult cases where the referring pathologists are uncertain of the diagnosis, recognize the inherent challenges of the case and regard "second opinion" part of their required/expected diligence in working up and finalizing a diagnosis. Other reasons include the lack of access to required laboratory investigations, tests, or molecular testing to confirm a diagnosis. "Second opinion" may be retrospective when there is an institutional review of outside pathology slides as a standard protocol for referral patients before definitive treatment. A retrospective request for "second opinion" can also be made directly by the patient, relatives or legal representatives. This may occur after investigation into the diagnosis by the patient and is becoming more common with the universal availability of the internet, lay access to medical literature and litigation. Finally, "second opinion" can be retrospectively requested when departmental audit or quality assurance reveals a disagreement between pathologists that cannot be resolved internally. "Second opinion" can account for a significant component of a specialist head and neck/oral and maxillofacial pathologist's workload. The number of "second opinion" evaluations should be audited when assessing target workloads for individual pathologists.

\section{Reject, endorse or enforce "double reporting"?}

Although a priori beneficial to the management of patients, "double reporting" and "second opinion" may be influenced by logistical and other factors, including costs from manpower, time and consumable resources.
Such costs are certainly measurable for formal second referral, but these data are largely unavailable for "double reporting".

More subtle and less measurable reasons why "double reporting" may not be widely practiced could include interpersonal issues, and perhaps (rarely) a negative attitude towards "having one's work checked", or practical issues such as turnaround time delays that might occur with this practice. Voluntary double reporting would appear to be good practice, and is probably commonplace in most diagnostic units. Most pathologists develop a particular interest and knowledge in specific organs or systems, and in many departments, this sub-specialization is encouraged and the diagnostic workload organized so each pathologist primarily reports cases within his (her) sphere of interest. Even when cases are not assigned to "specialists", pathologists tend to seek the advice of colleagues when faced with a difficult case outside their area of particular interest or knowledge.

Double reporting, especially mandatory double reporting of malignancies, may be criticized as time-consuming and/or unnecessary when a diagnosis is straightforward. For example, it is unlikely that a second pair of eyes is needed for a moderately differentiated squamous cell carcinoma (SCC) that invades submucosal skeletal muscle. It may, however, be helpful in a case of a very well-differentiated squamoproliferative lesion without submucosal invasion [3]. Selecting subsets of notoriously difficult or challenging cases for double reporting may be a useful compromise to help alleviate potential turnaround time delays. Further, double reporting may provide additional consistency in reporting, as both pathologists agree on the terminology to use and thereby standardize their diagnostic nomenclature. This practice is supported by the United States military services, where all malignancies are mandatorily prospectively double read to enhance diagnostic fidelity and patient management.

\section{Overview of "second opinion"}

Although there are no guidelines for identifying cases that should be sent for second opinion, auditing of pathology reports uncovers discrepancies across all major organ systems and shows some body sites and some lesions which are more prone to yield errors [4-6]. Site specific studies have shown that the head and neck area is a highrisk site, right behind the female reproductive tract and the gastrointestinal tract [7, 8]. Within head and neck areas, the thyroid gland is an error prone site [7], although the establishment of this organ as a specific specialty along with increased awareness has resulted in improvements. 
Excluding the nature of the referred cases, further exploration of the significance of "second opinion" requires awareness of the timing and routes of the requests, and the common reasons and difficulties precipitating a consultation. These are further examined below.

\section{Where to send second opinion cases?}

Although affected by idiosyncrasy, on the surface there should be no issues in determining the identity of the pathologist(s) to whom material should be referred for formal "second opinion": the obvious and easy choice being "experts" with a proven diagnostic experience and publication record in the field. The establishment of the specialty of head and neck and oral and maxillofacial pathology may have blurred the distinction between "expert" and "specialist". There are challenging issues related to defining an "expert" and assessing both the quality and quantity of publications. Defining what a "specialist" is, although desirable, has not been standardized in any national or international setting. Should designated specialists be individuals who have undergone special training in head and neck and/or oral and maxillofacial pathology or can a general pathologist who has a declared interest in reporting head and neck specimens also be considered a specialist? These questions remain to be resolved, but they certainly impact the decision regarding referral selection.

\section{When to request a "second opinion"?}

A second opinion can be requested before or after the case has been signed out.

Cases could be referred for "second opinion" after sign out when the treatment is followed by an unexpected clinical outcome. Clearly, retrospective second opinion can be fraught with potential problems, including a change in diagnosis potentially resulting in significant changes in patient management. The impact of a changed diagnosis cannot be easily measured and ramifications have rarely been systematically examined by clinicians or pathologists. Identifying discrepancies retrospectively can also have implications about the quality assurance and training needs of the original pathologist.

Accordingly and, to reduce the risk of diagnostic error leading to inappropriate management and possibly resulting in disability, suffering and death [9], a "second opinion" is more ideally requested from a specialist head and neck/oral and maxillofacial pathologist prior to signing out the definitive final diagnosis. Issuing a preliminary report or having a personal conversation with the treating clinician is generally considered good practice. Providing interim or provisional information to the clinician can help them to understand the reasons for the delay in reaching a final diagnosis, to plan future patient appointments and to keep the patient informed.

Medical ethics and standard accreditation guidelines dictate that a final diagnosis should be timely [8] and hence, an external referral case should be dispatched with and given the same priority as a routine internal case by the consultant pathologist. Nevertheless, the inherent degree of difficulty of these select cases, along with the frequent need to undertake further special studies and possible literature review will certainly affect reporting time. It is the obligation of the referring physician to send complete demographic information, pertinent clinical, laboratory and imaging findings, all of the histology slides and any special studies already performed, along with a representative tissue block or material for further investigation, if necessary and required for the diagnosis.

When "second opinion" is sought before the final diagnosis is issued, the potential benefits of preventing errors overall will almost always outweigh the potential time impact on the patient. Although generally minor, delay in diagnosis while awaiting the "second opinion" can result in heightened anxiety, uncertainty and inconvenience for the patient. Careful explanation of the reasons for the delay helps the clinician and patient and can greatly reduce frustration.

\section{Issuing "second opinion": practices and experiences}

Many head and neck surgical oncology departments have a protocol whereby the histologic slides from the original diagnostic material are reviewed by the local pathologist or specialist. This routine protocol is regarded as an important quality assurance practice potentially exposing diagnostic errors prior to definitive management [9, 10]. Ideally, outside pathology slides and reports should be reviewed for all patients referred to a specialist head and neck surgery unit [11] since there is evidence to suggest that a substantial number of head and neck lesions are misdiagnosed or miscategorized. Experience at the John Hopkins Cancer Centre [10] showed that, in a 10-year period, the outside diagnosis was changed, resulting in a significant modification in therapy or prognosis in $54(7 \%)$ of 814 cases. The majority of the changes (33 cases, $61 \%$ ) involved a change in tumor classification, but $13(24 \%)$ involved a change from a benign to a malignant diagnosis and eight $(15 \%)$ a change from a malignant to a benign diagnosis. This surprisingly high rate of discrepant diagnoses most likely reflects the difficult nature of many head and neck pathology diagnoses and the often complex categorization 
Table 1 Errors relating to oral/oropharyngeal pathology

Mistaking benign tangentially embedded squamous mucosal

biopsies for invasive well-differentiated SCC

Under or over diagnosing verrucous carcinoma

Mistaking papillary SCC for verrucous carcinoma

Mistaking pseudoepitheliomatous hyperplasia for SCC

Mistaking spindle cell carcinoma for sarcoma

Mistaking necrotizing sialometaplasia for SCC

Mistaking basaloid SCC for adenoid cystic carcinoma or neuroendocrine carcinoma

Failing to recognize adenosquamous carcinoma with a glandular component other than adenocarcinoma, NOS

Mistaking acantholytic SCC for adenosquamous carcinoma

Mistaking adenosquamous carcinoma for mucoepidermoid carcinoma

Mistaking small foci of tonsillar nonkeratinizing SCC as normal tonsillar crypts

Table 2 Errors relating to cervical lymph node pathology

Mistaking nodal metastatic HPV-positive non-keratinizing SCC of tonsil/base of tongue for a branchial cleft cyst or branchiogenic carcinoma

Mistaking nodal nevi for metastatic deposits

Mistaking salivary and rare thyroid glandular inclusions or inchoate nodal Warthin tumors for metastatic adenocarcinoma

Failure to recognize concurrent lymphoma

of salivary, sinonasal tract and odontogenic neoplasms. The reported rate of changed diagnosis in general surgical oncology pathology is lower at around 1.4-5.8 \% [4, 5, 12]. It would be most interesting to examine whether the rate of changed diagnoses has decreased in the decade since the John Hopkins experience was originally reported.

If, after reviewing the outside pathology slides, there is concurrence, it may seem unnecessary to issue a second report if there is no change in diagnosis. This may be the case for an incisional biopsy, when the archives of other hospitals are easily accessed. A second report on resections would be necessary if significant information for further management of the patient is lacking from the original report, such as the depth of invasion, margin status, or p16 immunohistochemistry for oropharyngeal carcinoma. Following the introduction and widespread endorsement of minimum datasets for histopathology reports, for example those recommended by the Royal College of Pathologists (United Kingdom) [13] or the Cancer Protocols from the College of American Pathologists, absence of such information seems unlikely. However, if the diagnosis was incorrect, this information may not have been reported (a benign diagnosis would not require a cancer reporting/ staging form). Even so, a second report should be issued for administrative reasons, collection of data and defining
Table 3 Errors relating to ear pathology

Mistaking aural polyp for extramedullary plasmacytoma or rhabdomyosarcoma

Mistaking SCC for "cholesteatoma"

Mistaking paraganglioma for carcinoid

Mistaking middle ear adenoma for ceruminous adenoma

Mistaking Langerhans cell histiocytosis for otitis media

Mistaking cholesterol granuloma for cholesteatoma

Mistaking accessory tragus for cutaneous papilloma

Mistaking first branchial arch anomalies for epidermal inclusion cysts

Mistaking otitis media with glandular metaplasia for middle ear adenoma

Table 4 Errors relating to sinonasal and nasopharyngeal pathology

Mistaking respiratory epithelial adenomatoid hamartoma for inverted Schneiderian papilloma

Mistaking reactive stromal atypia in sinonasal polyps for rhabdomyosarcoma

Failure to recognize allergic fungal sinusitis

Mistaking nasal turbinate for hemangioma

Mistaking undifferentiated nasopharyngeal carcinoma for lymphoma

Mistaking papillary adenocarcinoma for papillary rhinosinusitis

Mistaking inverted papilloma for SCC

Mistaking nasopharyngeal angiofibroma for fibrosed nasal polyp

Mistaking antrochoanal polyp for nasopharyngeal angiofibroma

Mistaking sinus mucocele for normal tissue

Mistaking lobular capillary hemangioma for glomangiopericytoma

Mistaking exophytic papilloma for cutaneous papilloma of nasal vestibule

workload. Common sense and professional courtesy are paramount. When in agreement, the second report could be brief, standardized or synoptic, using "I am in agreement with the diagnosis of the original report", although highlighting any additional information which may not have been included or reported specifically in the original report. If accurate, the additional information can be "replicated" from the original report and the contribution of the original pathologist acknowledged.

\section{Potential areas of misinterpretation}

There is a range of explanations for misdiagnosis. Examples from our experience are summarized in Tables 1, 2, 3, $4,5,6,7,8$ and 9 , with some previously discussed $[3,14$ 17]. Mistakes related to mucocutaneous melanocytic lesions are beyond the scope of this editorial and not further discussed. 
Table 5 Errors related to hypopharyngeal and laryngeal pathology

Mistaking hyaline stage of vocal cord polyp for amyloid

Mistaking vascular stage of vocal cord polyp for hemangioma

Mistaking fibrous stage of vocal cord polyp for neurofibroma

Mistaking myxoid stage of vocal cord polyp for myxoma

Mistaking Teflon injection for gout

Mistaking verruca vulgaris for verrucous SCC

Mistaking papillary keratosis for verrucous SCC

Mistaking papillary SCC for conventional SCC

Mistaking granular cell tumor with pseudoepitheliomatous hyperplasia for SCC

Mistaking spindle cell carcinoma for reactive changes or fibrosarcoma

Mistaking adenoid cystic carcinoma for neuroendocrine tumor

Mistaking typical carcinoid for atypical carcinoid

Mistaking small cell neuroendocrine carcinoma for undifferentiated carcinoma

Mistaking paraganglioma for atypical carcinoid

Mistaking small cell neuroendocrine carcinoma for lymphoma

Mistaking small cell neuroendocrine carcinoma for basaloid SCC

Mistaking chondrosarcoma for chondroma

Traditional traps of benign lesions mimicking oral SCC (such as pseudoepitheliomatous hyperplasia in chronic hyperplastic candidiasis or overlying a granular cell tumor and squamous islands in necrotizing sialometaplasia); mistaking cholesterol granuloma for cholesteatoma of the ear; and controversies (basal cell adenoma versus monomorphic adenoma) are more likely to be mentioned and reiterated in training [3]. Similarly, increased familiarity with the presentation and pattern of cervical metastases from oropharyngeal SCC is expected to reduce mistaking cystic metastasis for branchial cyst and debunk the myth of "branchial carcinoma" [14].

Borderline invasive lesions and mild or moderate squamous epithelial dysplasias are difficult to diagnose or categorize. The lack of a universally adopted system for classifying the borderline and superficially invasive SCC within the oral cavity and oropharynx accounts for some diagnostic discrepancies. While we have suggested a system for classifying borderline and superficially invasive mucosal cancers $[3,16]$, debate continues about the "best" system for grading squamous epithelial dysplasias, given the problems of: (1) inter- and intra- observer variability; (2) variability within lesions; and (3) progression or regression of lesions on successive biopsies [16, 18, 19]. Assessing malignant transformation of lichen planus and lichenoid dysplasia are typical problems.

Whether requests for "second opinion" originate from general pathologists or, less frequently, other specialist head and neck/oral and maxillofacial pathologists, influence how the consultant resolves the diagnostic challenges.
Table 6 Errors relating to salivary gland pathology

Mistaking multiple canalicular adenomata for invasive growth

Mistaking multifocal recurrent pleomorphic adenoma for carcinoma ex PA

Mistaking florid sialadenoma papilliferum for mucoepidermoid carcinoma

Mistaking polymorphous low-grade adenocarcinoma for pleomorphic adenoma

Mistaking epithelial-myoepithelial carcinoma for pleomorphic adenoma or metastatic renal cell carcinoma

Failure to recognize basal cell adenocarcinoma

Mistaking acinic cell carcinoma for adenoma

Mistaking necrotizing sialometaplasia for low-grade mucoepidermoid carcinoma

Mistaking oncocytic hyperplasia for oncocytoma

Failure to recognize variants of "myoepithelial" neoplasms and how to distinguish whether they are benign or malignant

Failure to recognize sclerosing adenosis and other variants of salivary hyperplastic lesions

Failure to characterize salivary tumors with a dominant clear-cell component

Mistaking Warthin's tumor for oncocytic papillary cystadenoma

Mistaking adenosquamous carcinoma for mucoepidermoid carcinoma

Mistaking spindle cell myoepithelioma for neurilemmoma

Mistaking extraglandular Warthin's tumor for metastatic carcinoma

Mistaking plasmacytoid myoepithelioma for extramedullary plasmacytoma

Mistaking adenoid cystic carcinoma for ameloblastoma, basal cell adenoma or pleomorphic adenoma

Mistaking benign lymphoepithelial lesion for malignant lymphoepithelial lesion/lymphoma

Failure to recognized highly hyalinized, benign lymphoepithelial lesion

Mistaking membranous basal cell adenoma for adenoid cystic carcinoma

Mistaking basal cell adenoma for pleomorphic adenoma

Mistaking granulomatous reaction to extravasated mucus for sarcoidosis or other granulomatous disease

Table 7 Errors relating to odontogenic pathology

Mistaking enlarged/hyperplastic dental follicle for dentigerous cyst or odontogenic fibroma

Mistaking dental papilla for odontogenic myxoma

Mistaking ameloblastoma of the sinonasal tract for adenoid cystic carcinoma

Failure to recognize aggressive odontogenic cysts such as glandular odontogenic cyst and variants of keratocystic odontogenic tumor (odontogenic keratocyst)

Mistaking nuclear pleomorphism in calcifying epithelial odontogenic tumor as malignancy 
Table 8 Errors relating to bone pathology

Confusing fibrous dysplasia, ossifying fibroma and periapical cemento-osseous dysplasia

Confusing chondrometaplasia, chondroma and low-grade chondrosarcoma

Failure to recognize parosteal osteosarcoma

Table 9 Errors relating to thyroid and parathyroid gland pathology

Mistaking follicular variant of papillary carcinoma for follicular adenoma

Mistaking hyalinizing trabecular tumor and follicular adenoma

Failure to recognize lymphoma against a background of chronic lymphocytic thyroiditis

Mistaking undifferentiated (anaplastic) thyroid carcinoma for sarcoma

Failure to recognize benign follicular adenoma in multinodular goiter

Failure to recognize mixed medullary and follicular (or papillary) carcinomas

Mistaking metastatic renal carcinoma for parathyroid carcinoma

A general pathologist may offer a differential diagnosis, or have specific questions related to histological, histochemical and immunohistochemical features or request advice on management and/or prognosis. Usually easy to resolve are requests from inexperienced pathologists or those unaware of particular histological features of the head and neck/oral and maxillofacial region. Enlarged dental follicles or the dental papilla of a supernumerary tooth are one of the most common cases referred with a provisional diagnosis of dentigerous cyst, odontogenic myxoma or odontogenic fibroma [20, 21]. Additional examples include the misinterpretation of the juxtaoral organ of Chievitz and subgemmal neurogenous plaques or other features associated with lingual foliate papillae/tonsil [3, 22]. Focal obstructive adenitis, a common feature in aging salivary glands, can be confused with intercalary duct lesions [23, 24]; the squamous metaplastic "feeder" salivary duct in a mucous extravasation cyst (mucocele) can be misinterpreted; and transepithelial elimination of sequestrae, not unusual in osteomyelitis, oral oncology patients with osteoradionecrosis and patients on bisphosphonates, can cause consternation when seen by general pathologists.

Also relatively straight forward are referrals regarding characteristic odontogenic lesions by general pathologists who are inexperienced with odontoma, ameloblastic fibroodontoma, dentigerous cyst and/or keratocystic odontogenic tumor, especially if they are inflamed or only partially sampled [15]. Non-specialists can be overwhelmed and frustrated by subtle gradients of cellularity and architecture in various salivary gland tumors (Table 6). Misdiagnosis of acinic cell carcinoma composed of non-serous cells showing cytoplasmic lumina and/or vacuoles, in various architectural arrangements, is another typical example [25]. Re-assessing labial salivary gland biopsies for focus scoring in possible Sjögren patients may be requested by ENT physicians or rheumatologists. Moreover, there may be concern about the precise characterization of a fibro-osseous lesion within the jaws (Table 8).

A less common, though far more interesting and challenging route for a second opinion is from another head and neck/oral and maxillofacial pathologist when cases may be referred for diagnosis, reassurance and/or interest. Obscure odontogenic lesions and salivary gland tumors are the main categories of such referrals, including rare odontogenic carcinomas and sarcomas [26] and unusual or hybrid salivary gland lesions (Table 6) [25]. Unusual or rare types of carcinomas may be referred to a more experienced colleague or one with a research interest, such as carcinoma cuniculatum; teratocarcinosarcoma, undifferentiated or other more recently characterized tumors (molecular profile specific) (Table 1) [3, 17].

The diagnostic difficulties may prove difficult to overcome despite extensive investigations and discussion between experts or specialists. Experience with head and neck histopathology National External Quality Assessment Schemes [27] shows cases characterized as "Educational", have widely different opinions distributed among a wide number of participants. In such instances, the issued final report should: (1) explain the reasons for not reaching a definite diagnosis; (2) present the arguments for and against the different possibilities; and (3) advise on further management. For example, when extensive discussion on a palatal tumor fails to distinguish between minimally invasive carcinoma ex pleomorphic adenoma; epithelialmyoepithelial carcinoma; and pleomorphic adenoma showing calcification of hyalinized stroma and biphasic pattern with adluminal clear cells, the clinician would benefit from the pathologist's understanding of the histological grade and expected outcome for those entities.

Dealing with discrepant diagnoses uncovered during review on the patients' referral to a specialist can be difficult [28, 29]. As a matter of professional courtesy, the original pathologist should be contacted and informed of the reason for the change and whether additional procedures were carried out, ideally before the amended report is issued. Discovering a serious error may precipitate remedial or quality assurance action towards the original pathologist. Targeted education or retraining may be advised, realizing the possible negative consequences the original pathologist may experience.

\section{How should "second opinion" be funded?}

This is a challenging issue and the way this should be addressed depends on the local situation. Each country has 
a differently financed healthcare system-hence, the problem of ensuring specialized consultation in difficult cases is ubiquitous. In an ideal world, for the sake of patient safety, second opinions would be free of charge even though there is an obvious cost. Some systems, such as the United States and Canada, reimburse for "second opinion" cases, while other countries regard them as part of quality assurance without charge. Still other pathology departments have operated on a reciprocal arrangement. However, with growing compliance and auditing restrictions, "free" or "barter" arrangements are being replaced by a tariff system based on pathological workload codes (such as CPT codes). Charging a fee may even be counterproductive if a patient is referred with an incomplete pathology report and the surgeon at the referral center opts to repeat the diagnostic biopsy with a repeat of the full diagnostic workup. The real cost in terms of manpower and consumables/resources varies depending on the nature of the referred material (unprocessed specimen, processed tissue block, unstained slides, routinely stained slides, range of special stains including immunohistochemistry), the number of tissue blocks and slides, and the diagnostic difficulty. Telepathology may provide a way of reducing the time and cost of a second opinion [30]. Many consultant pathologists prefer to evaluate difficult cases by physical rather than virtual microscopic slides, although this preference will probably change in time.

\section{Conclusions}

Second opinion pathology consultation occurs in a variety of different clinical settings and with different driving factors. Some areas of head and neck pathology are fraught with potential error or controversy and these are frequent sources of second opinion consultations. Ideally, these second opinions should be performed prospectively and in real-time before treatment is undertaken. Retrospective second opinions are frequent in head and neck pathology. Discrepancies should be handled professionally and promptly so the patient receives optimal care.

\section{References}

1. Kornstein MJ, Byrne SP (2007) The medicolegal aspect of error in pathology: a search of jury verdicts and settlements. Arch Pathol Lab Med 131:615-618

2. Nakhleh RE, Bekeris LG, Souers RJ, Meier FA, Tworek JA (2010) Surgical pathology case reviews before sign-out: a College of American Pathologists Q-Probes study of 45 laboratories. Arch Pathol Lab Med 134:740-743
3. Woolgar JA, Triantafyllou A (2009) Pitfalls and procedures in the histopathological diagnosis of oral and oropharyngeal squamous cell carcinoma and a review of the role of pathology in prognosis. Oral Oncol 45:361-385

4. Kronz JD, Westra WH, Epstein JI (1999) Mandatory second opinion surgical pathology at a large referral hospital. Cancer $86: 2426-2435$

5. Tsung JS (2004) Institutional pathology consultation. Am J Surg Pathol 28:399-402

6. Troxel DB (2003) Pitfalls in the diagnosis of malignant melanoma: findings of a risk management panel study. Am J Surg Pathol 27:1278-1283

7. Kronz JD, Westra WH (2005) The role of second opinion pathology in the management of lesions of the head and neck. Curr Opin Otolaryngol Head Neck Surg 13:81-84

8. Woolgar JA, Ferlito A, Devaney KO, Rinaldo A, Barnes L (2011) How trustworthy is a diagnosis in head and neck surgical pathology? Eur Arch Otorhinolaryngol 268:643-651

9. Jones K, Jordan RC (2010) Patterns of second-opinion diagnosis in oral and maxillofacial pathology. Oral Surg Oral Med Oral Pathol Oral Radiol Endod 109:865-869

10. Westra WH, Kronz JD, Eisele DW (2002) The impact of second opinion surgical pathology on the practice of head and neck surgery: a decade experience at a large referral hospital. Head Neck 24:684-693

11. Association of Directors of Anatomic and Surgical Pathology (1993) Consultations in surgical pathology. Am J Surg Pathol 17:743-745

12. Abt AB, Abt LG, Olt GJ (1995) The effect of interinstitution anatomic pathology consultation on patient care. Arch Pathol Lab Med 119:514-517

13. Helliwell T, Woolgar JA (2011) Royal College of Pathologists standards and datasets for reporting cancers. Royal College of Pathologists, London

14. Woolgar JA, Triantafyllou A (2010) Lymph node metastases in head and neck malignancies: assessment in practice and prognostic importance. Diagn Histopathol 16:265-275

15. Woolgar JA, Triantafyllou A (2010) Deviant odontogenic tissues. Diagn Histopathol 16:538-544

16. Woolgar JA, Triantafyllou A (2011) Squamous cell carcinoma and precursor lesions: clinical pathology. Periodontol 2000 $57: 1-22$

17. Slootweg PJ, Ferlito A, Cardesa A, Thompson LD, Hunt JL, Strojan P, Takes RP, Triantafyllou A, Woolgar JA, Rinaldo A, Devaney KO, Barnes L (2013) Sinonasal tumors: a clinicopathologic update of selected tumors. Eur Arch Otorhinolaryngol 270:5-20

18. Warnakulasuriya S (2001) Histological grading of epithelial dysplasia: revisited. J Pathol 194:294-297

19. Ho MW, Field EA, Field JK, Risk JM, Rajlawat BP, Rogers SN, Steele JC, Triantafyllou A, Woolgar JA, Lowe D, Shaw RJ (2013) Outcomes of oral squamous cell carcinoma arising from oral epithelial dysplasia: rationale for monitoring premalignant oral lesions in a multidisciplinary clinic. Br J Oral Maxillofac Surg 51:594-599

20. Kim J, Ellis GL (1993) Dental follicular tissue: misinterpretation as odontogenic tumors. J Oral Maxillofac Surg 51:762-767 (discussion 767-768)

21. Hoffman S, Jacoway JR, Krolls SO (1987) Intraosseous and parosteal tumors of the jaws. In: Hartmann WH, Sobin LH (eds) Atlas of tumor pathology, second series, fascicle 24. Armed Forces Institute of Pathology, Washington, DC, pp 203-209

22. Triantafyllou A, Coulter P (2004) Structural organization of subgemmal neurogenous plaques in foliate papillae of tongue. Hum Pathol 35:991-999 
23. Triantafyllou A, Hunt JL, Devaney KO, Ferlito A (2013) A perspective of comparative salivary and breast pathology. Part I: microstructural aspects, adaptations and cellular events. Eur Arch Otorhinolaryngol [Epub ahead of print]

24. Weinreb I, Seethala RR, Hunt JL, Chetty R, Dardick I, PerezOrdoñez B (2009) Intercalated duct lesions of salivary gland. A morphological spectrum from hyperplasia to adenoma. Am $\mathbf{J}$ Surg Pathol 33:1322-1329

25. Chiosea SI, Peel R, Barnes EL, Seethala RR (2009) Salivary type tumors seen in consultation. Virchows Arch 454:457-466

26. Woolgar JA, Triantafyllou A, Ferlito A, Devaney KO, Lewis JS Jr, Rinaldo A, Slootweg PJ, Barnes L (2013) Intraosseous carcinoma of the jaws: a clinicopathologic review. Part II: odontogenic carcinomas. Head Neck 35:902-905

27. Woolgar JA (2006) The national head and neck histopathology external quality assurance scheme: evolution, design and analysis of 11 circulations from 1999 to 2005. J Clin Pathol 59:482-488

28. Manion E, Cohen MB, Weydert J (2008) Mandatory second opinion in surgical pathology referral material: clinical consequences of major disagreements. Am J Surg Pathol 32:732-737

29. Swapp RE, Aubry MC, Salomão DR, Cheville JC (2013) Outside case review of surgical pathology for referred patients: the impact on patient care. Arch Pathol Lab Med 137:233-240

30. Etit D, Tan A, Bakir K, Cakalagaoglu F, Elagoz S, Elpek GO, Han O, Han U, Hucumenoglu S, Koybasioglu F, Kucuk U, Kulacoglu S, Paker I, Sarioglu S, Seckin S, Tekkesin MS, Uguz A, Unal T, Gunhan O (2013) Interobserver agreement in salivary gland neoplasms by telepathy: an analysis of 47 cases. Anal Quant Cytol Histol 35:114-120 